\title{
Massive hemoptysis in a patient with chronic pancreatitis
}

V. Vimalraj, MS, ${ }^{\text {a }}$ R. Surendran, MCh, ${ }^{a}$ K. S. Sekar, MD, ${ }^{b}$ and N. Rajendran, DM, ${ }^{\mathrm{c}}$ Chennai, India

Additional figures are available online. $\mathcal{B}$

From the Departments of Surgical Gastroenterology, ${ }^{a}$ Government Stanley Medical College and Hospital, Chennai, India, and the Departments of Radiology ${ }^{\mathrm{b}}$ and Medical Gastroenterology, ${ }^{\mathrm{c}}$ Apollo Hospital, Chennai, India.

Received for publication March 1, 2005; accepted for publication March 28, 2005.

Address for reprints: Velayutham Vimalraj, Department of Surgical Gastroenterology, Government Stanley Medical College, Oldjail Rd, Royapuram, Chennai, Tamilnadu 600001, India (E-mail: drvimmi@ @ediffmail.com).

J Thorac Cardiovasc Surg 2005;130:910-1

$0022-5223 / \$ 30.00$

Copyright $\odot 2005$ by The American Association for Thoracic Surgery

doi:10.1016/j.jtcvs.2005.03.033
H emoptysis caused chronic pancreatitis is rare. It could be a result of various causes. Management depends

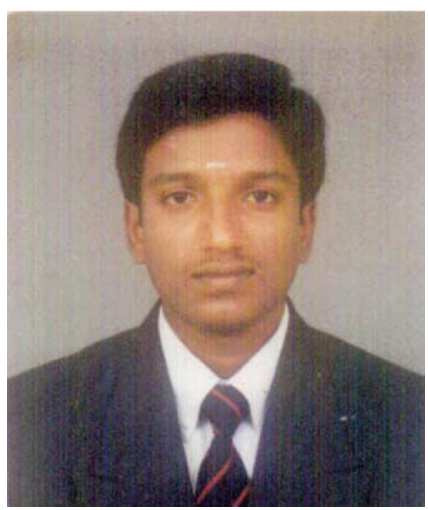

Dr Vimalraj on the cause. Our patient, who had history of chronic calcific pancreatitis, underwent pancreatic stenting and extracorporeal shock wave lithotripsy (ESWL), after which the patient had massive hemoptysis and hematochezia necessitating massive blood transfusion, which was found to be due to a pseudoaneurysm of the splenic artery eroding into the left lung and colon. The patient underwent surgical intervention for correction of the problem.

\section{Clinical Summary}

A 43-year-old man was admitted to our hospital in May 2004. He had abdominal pain radiating to the back on and off for 2 years, 4 to 5 episodes per year. This was intractable pain requiring admission and medical treatment in different hospitals.

Abdominal computed tomographic scanning during admission showed multiple stones in the pancreas, with a dilated main pancreatic duct containing multiple stones from head to tail. The patient was advised to undergo surgical intervention. He was not willing to undergo such intervention and was subjected to pancreatic stenting, followed by ESWL. The patient's condition was apparently normal for 2 days, but on the third day, he had severe abdominal pain, which was managed medically. The abdominal pain persisted and was conservatively managed, and the patient had massive hemoptysis, requiring 8 units of blood transfusion for resuscitation.

During admission to our hospital in June 2004, the patient provided a history of severe pain of the abdomen. Examination showed that the patient was pale, with a temperature of $37^{\circ} \mathrm{C}$, a pulse rate of 100 beats $/ \mathrm{min}$, a blood pressure of $100 / 70 \mathrm{~mm} \mathrm{Hg}$, and a respiratory rate of 22 breaths/min. The left lung was dull on percussion, and the breath sounds were diminished on the left side. Laboratory values were as follows: a hemoglobin value of $4 \mathrm{~g} / \mathrm{dL}$, a hematocrit value of $16.4 \%$, bleeding time and clotting time within normal limits, and a serum amylase concentration of 1253 $\mathrm{U} / \mathrm{L}$. Various pulmonary causes of hemoptysis were ruled out, and polymerase chain reaction qualitative assay for tuberculosis was done, the result of which was negative. On the 10th day, the patient experienced hematochezia.

The patient underwent an angiogram, which showed a pseudoaneurysm of the splenic artery. The pseudoaneurysm was communicating with the left lung. Embolization of the pseudoaneurysm was attempted, but it failed (Figure 1). 


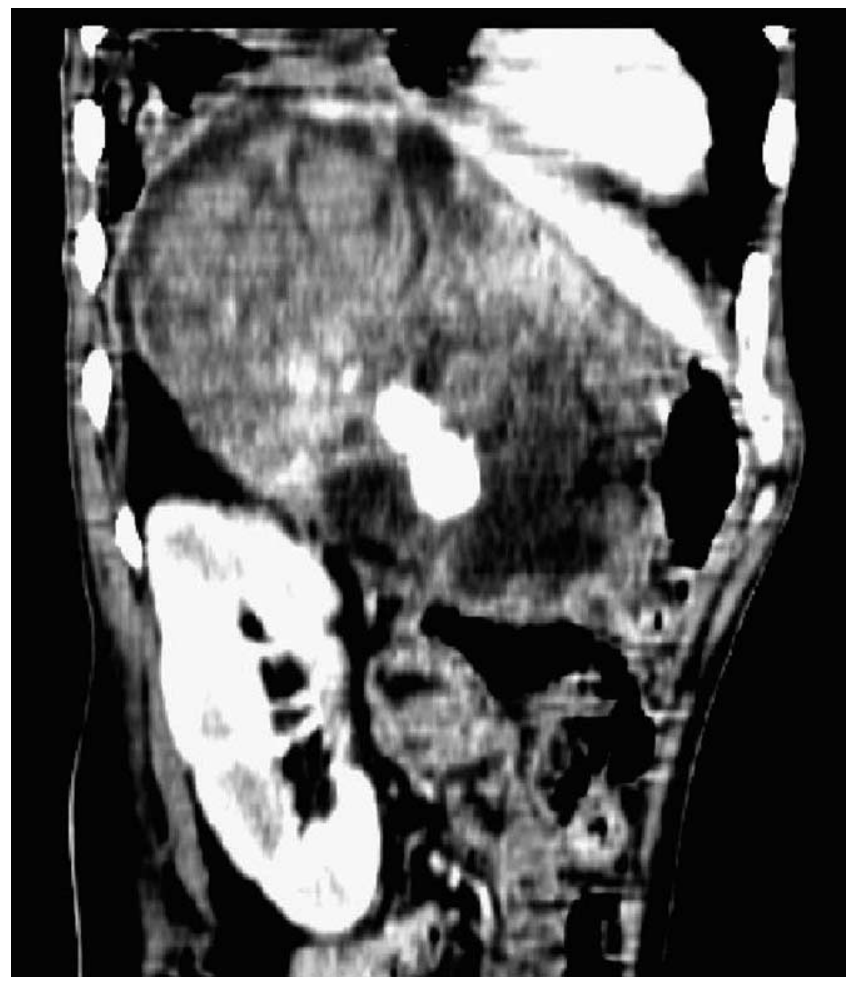

Figure 1. Abdominal computed tomographic scan showing splenic artery pseudoaneurysm.

The patient had hematochezia and underwent an emergency operation, the findings of which were consistent with those of the angiogram: a $6 \times 6$-cm pseudoaneurysm was seen that communicated with the left lung through a rent in the diaphragm, and it also communicated with the colon, which resulted in hematochezia.

Excision of the distal pancreas along with the aneurysm, splenectomy, closure of the rent in the diaphragm, resection of the affected colon, and a temporary colostomy were performed. The left side of the chest was drained with an intercostal drain. The patient had an uneventful postoperative period and recovered well (Figure 2 and Figures E1-E5).

\section{Discussion}

Pleuropulmonary complications of chronic pancreatitis are many and nonspecific. They can present as pleural effusion, basal atelectasis, pleural reaction, or a raised hemidiaphragm. Hemoptysis caused by chronic pancreatitis rarely has been reported and has always required pancreatic surgical intervention. Hemoptysis in chronic pancreatitis might be due to congestion of the left lung or formation of a pleuropulmonary fistula or due to rupture of a pseudoaneurysm of the splenic artery into the left lung. ${ }^{1}$

The potential application of endoscopic treatment is limited to a subgroup of patients with amenable pancreatic ductal anatomy. These are patients with dilated pancreatic ducts who also have a single dominant stricture or obstructing stone in the head of the pancreas, with dilation of the duct upstream of the stone or

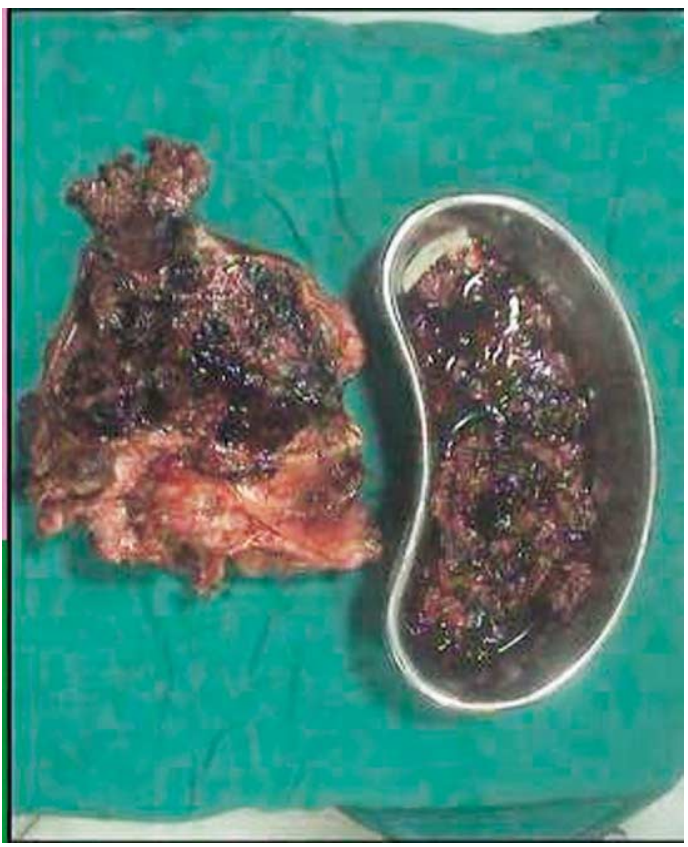

Figure 2. Pseudoaneurysm: resected specimen.

stricture. Strictures and calculi in the tail of the pancreas are not amenable to endoscopic treatment. ${ }^{2}$

Although ESWL can be delivered safely with good efficacy, it is associated with various complications. Acute abdominal symptoms, a significant increase in the white blood cell count, alanine aminotransferase level, and bilirubin level, and exacerbation of pancreatitis are commonly reported. Rare complications, such as subcapsular hematoma of the liver, splenic abscess, splenic infarction, and rupture, are also reported. ${ }^{3-5}$ This is the first report of hemoptysis caused by rupture of the pseudoaneurysm of the splenic artery after ESWL and stenting.

Takanami ${ }^{1}$ has reported a case of massive hemoptysis complicating chronic pancreatitis because of communication between the inferior phrenic artery and the pulmonary artery. Metallic coils and gelatin sponge embolization of the inferior phrenic artery controlled the hemoptysis. In our patient, splenic artery embolization was tried with metallic coils, but it failed because of technical reasons.

In the present case, stenting and ESWL have created a scenario of acute pancreatitis, which resulted in a pseudoaneurysm that rapidly increased in size to erode into the left lung and colon, resulting in massive hemoptysis and hematochezia. In a good surgical candidate, it is better to avoid ESWL and stenting.

\section{References}

1. Takanami I. Massive haemoptysis due to chronic pancreatitis: control with inferior phrenic artery embolisation. Eur J Cardiothorac Surg. 2000;18:120-2.

2. Forsmark CE. Chronic pancreatitis. In: Feldman M, Friedman LS, Sleisenger MH, editors. Sleiseger and Fordtran's gastrointestinal and 
liver disease: pathophysiology/diagnosis/management. Vol 1. Philadelphia: Saunders. 2002; p. 958-9.

3. van der Hul R, Plaisier P, Jeekel J, Terpstra O, den Toom R, Bruining H. Extracorporeal shock-wave lithotripsy of pancreatic duct stones: immediate and long-term results. Endoscopy. 1994;26:573-8.

4. Hirata N, Kushida Y, Ohguri T, Wakasugi S, Kojima T, Fujita R. Hepatic sub capsular hematoma after extra corporeal lithotripsy (ESWL) for pancreatic stones. J Gastroenerol. 1999;34:713-6.

5. Plaisier PW, den Hoed PT. Splenic abscess after lithotripsy of pancreatic duct stones. Dig Surg. 2001;18:231-2. 


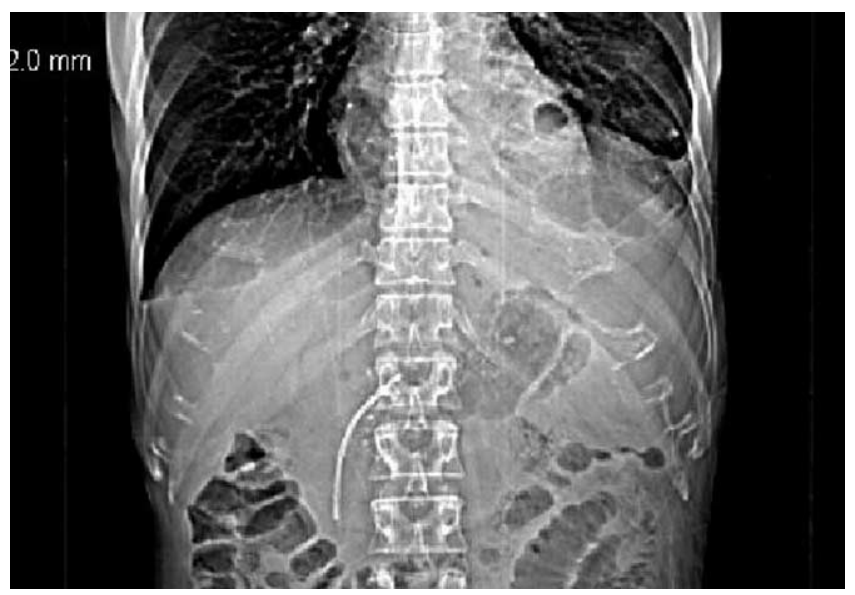

Figure E1. Scanogram showing pancreatic stent.

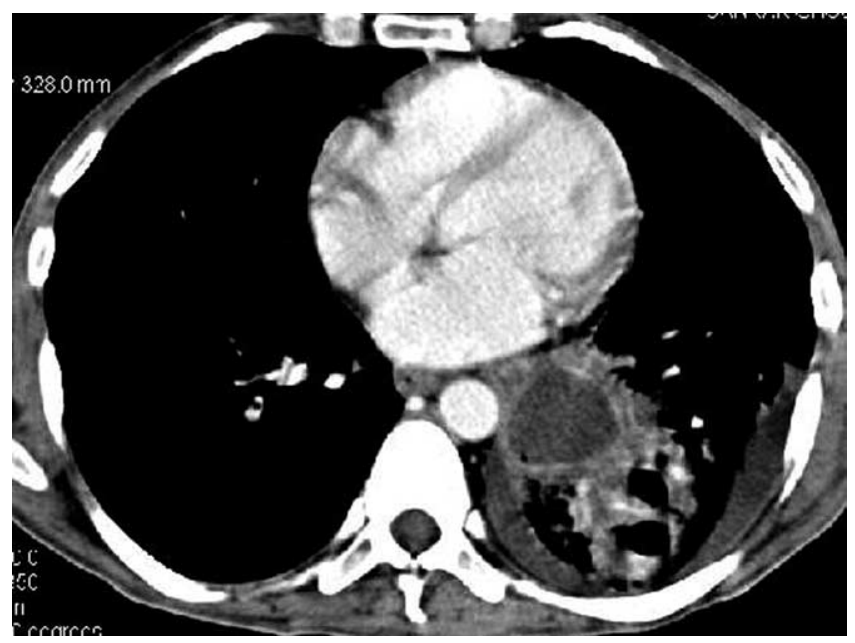

Figure E2. Computed tomographic scan of the chest (plain) showing communication with the bronchus.

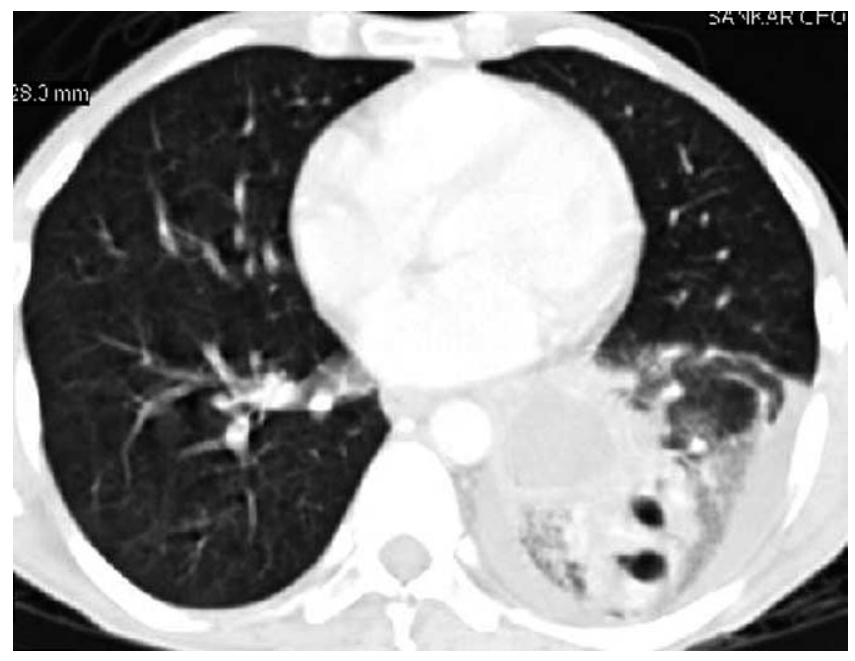

Figure E3. Computed tomographic scan of the chest (contrast) showing communication with the bronchus. 


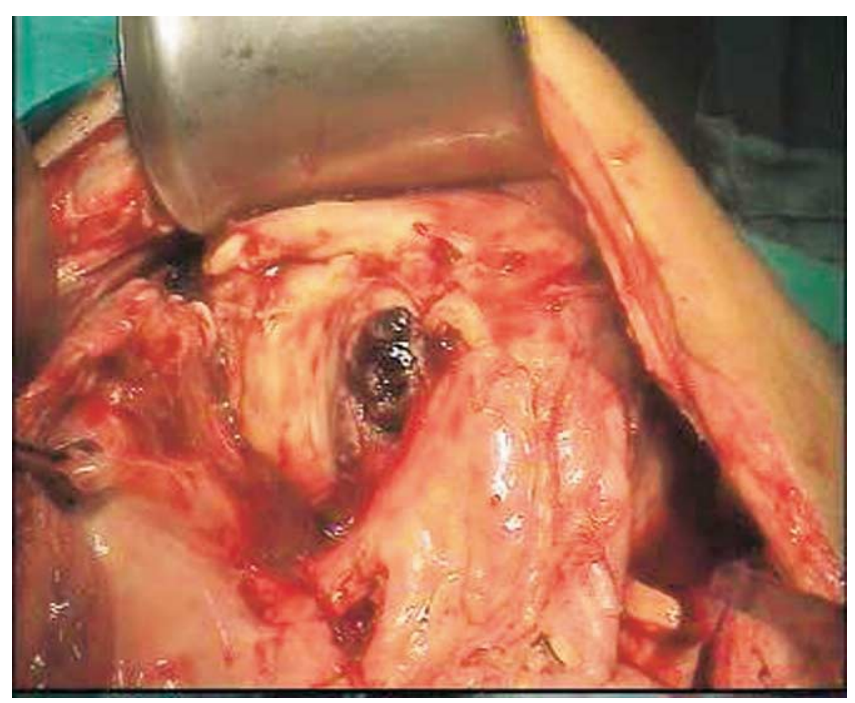

Figure E4. Pseudoaneurysm: before resection.

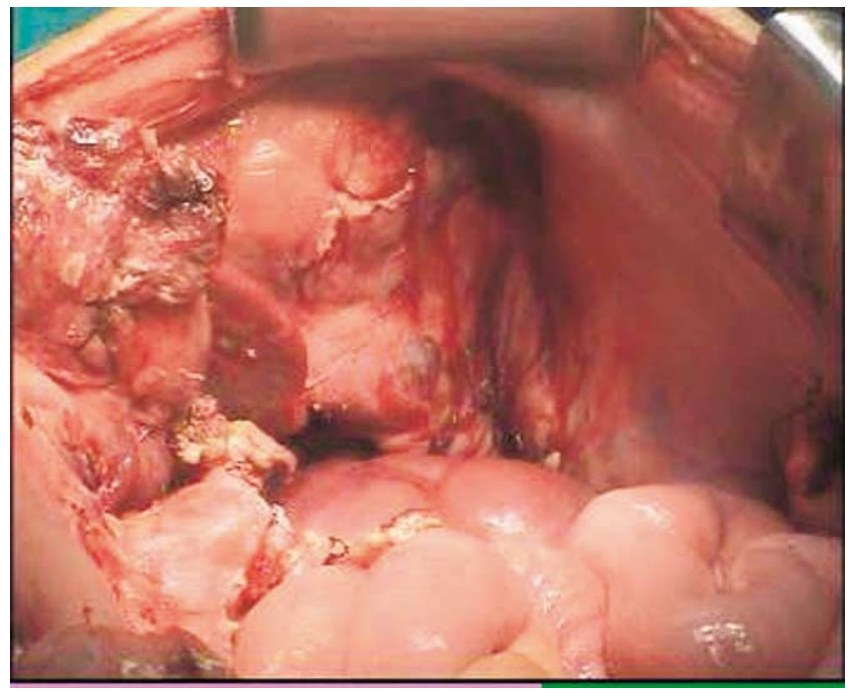

Figure E5. Pseudoaneurysm: after resection.

910.e5 The Journal of Thoracic and Cardiovascular Surgery • September 2005 